\title{
BRECHAS SALARIALES EN URUGUAY: GÉNERO, SEGREGACIÓN Y DESAJUSTES POR CALIFICACIÓN
}

\author{
Alma Espino* \\ Fecha de recepción: 30 de octubre de 2012. Fecha de aceptación: 25 de febrero de 2013.
}

\begin{abstract}
RESUMEN
Se analizan las diferencias salariales por sexo considerando el efecto de la segregación laboral y de los desajustes en las calificaciones laborales, por medio de la estimación de ecuaciones que incorporan estas variables explicativas. Los resultados indican que la segregación es fundamental para entender la persistencia de las brechas salariales entre trabajadores de ambos sexos, aunque una parte sustancial de éstas es atribuible al sexo de los individuos. Las variables tradicionales de capital humano prácticamente no explican la brecha salarial de género, mientras que los desajustes educativos en particular la sobre educación, también contribuye a dichas brechas. Todo ello tiene implicaciones para las políticas públicas, para valorar los avances por las mujeres rompiendo los estereotipos de género que conllevan a una marcada discriminación económica.
\end{abstract}

Palabras clave: segregación, brechas salariales, sobrecalificación, subcalificación.

\section{Salary Gaps in Uruguay: Gender, Segregation AND UNEQUAL LABOr QUALIFICATIONS}

\begin{abstract}
This text analyzes salary differences between genders, taking into account the effect of labor segregation and unequal labor qualifications, by estimating the equations that incorporate these explanatory variables. The results indicate that segregation is fundamental to understand the persistence of salary gaps among employees of both genders, although a substantial portion is attributable to the gender of individuals. Traditional human capital variables do not really explain the gender salary gap, while educational inequality is another contributing factor. All of this has broad implications for public policy, in order to evaluate women's advances in breaking the gender stereotypes implied by marked economic discrimination.
\end{abstract}

Key Words: Segregation, Salary Gaps, Over-Qualification, Under-Qualification.

\footnotetext{
* Investigadora del Instituto de Economía. Facultad de Ciencias Económicas y de Administración. Universidad de la República. Uruguay. Correo electrónico: alma@iecon.ccee.edu.uy
} 


\section{ÉCARTS DE SALAIRE EN URUGUAY : GENRE, SÉGRÉGATION ET DISPARITÉS DUES À LA QUALIFICATION \\ Résumé}

Il est procédé à l'analyse des différences de salaires entre les deux sexes en considérant l'effet de la ségrégation sur le plan professionnel et des disparités dans les qualifications professionnelles, au moyen de l'estimation d'équations qui englobent ces variables explicatives. Les résultats indiquent que la ségrégation est fondamentale pour comprendre la persistance des écarts de salaire entre les travailleurs des deux sexes, bien qu'une partie substantielle de ces écarts soit attribuable au sexe des individus. Les variables traditionnelles de capital humain n'expliquent pas dans la pratique l'écart de salaire entre sexes, tandis que les disparités éducatives notamment la sur-éducation, contribue aussi à les creuser. Tout cela a des implications pour les politiques publiques, pour valoriser les avancées des femmes en rompant les stéréotypes de genre qui mènent à une discrimination économique marquée.

Mots clés: Ségrégation, écarts de salaire, surqualification, sous-qualification.

\section{BRECHAS SALARIAIS NO URUGUAI: GÊNERO, SEGREGAÇÁO E DESAJUSTES POR QUALIFICAÇÃO}

\section{Resumo}

No presente trabalho se analisam as diferencias salariais por sexo, considerando o efeito da segregação laboral e dos desajustes nas qualificaçóes laborais, por meio da estimação de equaçóes que incorporam estas variáveis explicativas. Os resultados indicam que a segregação é fundamental para entender a persistência das brechas salariais entre trabalhadores de ambos os sexos, ainda que uma parte substancial destas seja atribuível ao sexo dos indivíduos. As variáveis tradicionais de capital humano praticamente não explicam a brecha salarial de gênero, enquanto que os desajustes educativos em particular sobre a educação, também contribui a ditas brechas. Tudo isso tem implicaçóes para as políticas publicas, para avaliar os avanços feitos pelas mulheres rompendo os estereótipos de gênero que levam a uma marcada descriminação econômica.

Palavras-chave: Segregação, brechas salariais, sobre-qualificação, sub-qualificação.

乌拉圭的工资差距：性别、隔离与不平等的劳工资质

摘要:

本文通过结合劳工隔离、不平等的劳工资质等解释性变量和建立估算平等式, 分析了工资因性别而产生的差异。研究结果表明, 劳工差异是解释两性员工之 间工资差异的基本变量，尽管很大一部分差异归因于个体的性别。传统的人力 资本变量不能真正解释因性别产生的工资差异, 而教育不平等是另外的解释变 量。这些研究结论对公共政策具有广泛的启示意义, 特别是在评估女性发展方 面, 以打破经济歧视在性别方面所形成的障碍。

关键词：隔离工资差距 资质过高资质不足 


\section{INTRODUCCIÓN}

En el mercado laboral uruguayo las mujeres presentan menores salarios que los hombres en promedio aunque las diferencias salariales por sexo tienden a disminuir coincidentemente con lo observado para otros países. La interpretación de las brechas salariales de género en el marco de la economía neoclásica suele basarse en diferencias de productividad así como en factores de discriminación. Esta última aparece cuando las diferencias en las remuneraciones por trabajo no se explican por las características económicas que afectan la productividad de los trabajadores, o por las características de los puestos de trabajo.

En un sentido más amplio, la discriminación hacia las mujeres se entiende como parte del proceso por el cual se adquieren las habilidades y las capacidades para ingresar al mercado laboral, y que luego contribuyen a las diferencias de género reales o percibidas en la productividad. Esto es, la discriminación en la educación, la contratación y/o la promoción en el empleo pueden aumentar los costos de adquisición de habilidades para el grupo discriminado y la remuneración que obtienen puede no compensar ese mayor costo. La formación de expectativas en ese marco puede actuar desalentando a los individuos con determinadas preferencias que no responden a las normas de género prevalecientes. Por tanto, los determinantes de la elección de la ocupación pueden ser considerados en parte endógenos. Las normas (formales e informales) y las expectativas del medio social son especialmente importantes para las economistas feministas en la formación de las preferencias de hombres y mujeres en términos de educación y empleo. Estas podrían subestimar sistemáticamente el valor, la habilidad y el esfuerzo que implican actividades construidas como "femeninas", en un orden social donde las mujeres son una categoría de género subordinado (Johansson y Katz, 2007).

En este trabajo, en primer lugar, se cuantifica la segregación laboral de género entre tipos de ocupación, ramas de actividad económica y establecimientos empresariales. Se analiza luego el desajuste presente en la calificación de la fuerza de trabajo uruguaya, así como la vinculación entre este fenómeno y la segregación ocupacional. Finalmente, se calcula la magnitud de la incidencia de la segregación laboral y los desajustes por calificación en las brechas salariales, por medio de la estimación de ecuaciones que incorporan estas variables explicativas. 


\section{FACTORES A CONSIDERAR: SEGREGACIÓN LABORAL Y DESAJUSTES POR CALIFICACIÓN}

\section{Segregación laboral}

La segregación ocupacional de género -entendida como la concentración desproporcionada de las mujeres- en relación con su participación en la fuerza de trabajo -en ciertas ocupaciones- ha sido recogida en la literatura como un factor que contribuye a las diferencias salariales entre hombres y mujeres. Numerosos trabajos empíricos procuran cuantificar la evolución de la segregación en el nivel de ocupaciones, industrias y establecimientos y su efecto sobre los salarios. ${ }^{1}$ En el caso uruguayo, la segregación ocupacional aparece como un fenómeno persistente y ha sido empíricamente identificada como una de las principales fuentes de las diferencias salariales entre los individuos de ambos sexos. Amarante y Espino (2004) analizan la incidencia de la segregación ocupacional en las diferencias salariales por sexo en el sector privado (1990-2000). Concluyen que las diferencias salariales entre hombres y mujeres obedecen a que los salarios de las mujeres son afectados negativamente por la concentración de mujeres en las ocupaciones. Sin embargo, los salarios de los hombres en Uruguay no se ven afectados a la baja por la inserción laboral en ocupaciones feminizadas. El trabajo confirma las diferencias en los retornos a las características económicas entre hombres y mujeres, y el efecto de la segregación ocupacional, cuya importancia además, aumenta en el periodo considerado. Esta aumenta las diferencias salariales, ya que implica una penalización por pertenecer a ocupaciones femeninas para las mujeres y no para los hombres.

\section{Desajustes en las calificaciones para el empleo}

Se entiende por desajustes por calificaciones (skill mismatch) a la existencia de sub o sobre calificación entre los trabajadores respecto a los puestos que ocupan, lo cual puede expresarse en la productividad laboral, el desempleo y las remuneraciones. Las calificaciones laborales se obtienen en el sistema formal y en el desarrollo y la acumulación de competencias profesionales en el lugar

1 Entre otros Bergmann (1974), Polachek (1979), Blau (1984), Johnson y Solon (1986), Cabral Vieira, Rute Cardoso, Portela (2003) Bernat Díaz (2009), Nacira María Barraza Narváez (2010). 
de trabajo o mediante la socialización. La calificación adecuada, la sobrecalificación o la subcalificación son conceptos relacionados con las características particulares de los puestos que se ocupan por lo cual, se trata de situaciones que pueden modificarse.

Los desajustes por calificación se han explicado desde diferentes perspectivas teóricas como la del capital humano, la "señalización", competencias por puestos de trabajo, búsqueda con información imperfecta, entre otras (Leuven y Oosterbeek, 2011).

El análisis de los desajustes por calificaciones y su efecto sobre los salarios ha sido examinado con modelos que incorporan la sobrecalificación y la subcalificación Over, Required, Under Education (ORU) para explicar las diferencias en las remuneraciones (Verdugo y Verdugo, 1989; Hartog, 2000; Groot y Maassen, 2000). Los resultados obtenidos muestran que los diferenciales salariales además de desfavorecer a quienes han alcanzado un nivel de educación formal superior al requerido en el empleo (sobrecalificados), suelen beneficiar a quienes tienen un nivel de educación inferior al demandado en su puesto de trabajo (subcalificados) (Cohn y Kahn, 1995; Allen y Van der Velden, 2001). Otros trabajos indican que los años de sobreeducación tienen una tasa de rendimiento positiva, aunque inferior a la de los años requeridos en el puesto de trabajo, mientras que los de infraeducación tienen una tasa de rendimiento negativa (Duncan y Hoffman, 1981; Hartog y Oosterbeek, 1988; Sicherman, 1991; Cohn y Ng, 2000; Daly, Büchel y Duncan, 2000; Groenelveld y Hartog, 2004).

Si bien las diferencias de género en educación han sido centrales en muchos análisis sobre las brechas salariales, es menos común que se consideren los desajustes por calificaciones con este propósito. ${ }^{2}$ En Uruguay el tema de los desajustes ha sido analizado recientemente por Espino (2011) encontrando que la probabilidad de estar sobrecalificado es mayor para las mujeres -más propensas a gozar de más años de educación formal que lo que se requiere normalmente para su actividad profesional- mientras que los hombres son propensos a la subcalificación. Esta situación se verifica en diferentes estudios (Hertz et al., 2008). Por su parte, en América Latina la subrepresentación en puestos gerenciales y la sobrerepresentación femenina en ocupaciones tales

2 El modelo neoclásico de especialización de los hogares se ha empleado para explicar por qué las mujeres tienen más probabilidades de estar sobre calificadas relacionándolo por ejemplo, con las opciones condicionadas por mercados particulares (Frank, 1978). Una discusión sobre esta perspectiva puede verse en Büchel y Battu (2002). 
como trabajadoras de servicios, personal administrativo, servicio doméstico y en ciertos sectores de actividad no estaría de acuerdo con sus niveles educativos (Ñopo, 2012).

Johansson y Katz (2007) investigan los desajustes educativos y su repercusión en las diferencias salariales de género y en los retornos a la educación en Suecia entre 1993 y 2002, con resultados similares respecto a las propensiones de mujeres y hombres. Las descomposiciones de la brecha salarial por sexo ajustando por el mismatch de calificaciones la disminuyen entre un décimo y un sexto, es decir, entre un tercio y la mitad de lo que contribuye la segregación por rama de actividad económica.

\section{METODOLOGÍA}

Con el propósito de medir la segregación ocupacional y su evolución se calcula el índice de Duncan; posteriormente, se estima la incidencia de la segregación en las brechas salariales con base en Bayard et al. (2003) incorporando además la corrección por sesgo de selección de Heckman. Finalmente, luego de estimar la probabilidad de encontrarse sobre o subcalificado se desarrollan regresiones salariales que toman en cuenta estos aspectos (ORU), considerando especialmente el papel que desempeñan en sus resultados la segregación laboral de género. También en este caso se descomponen los resultados para explicar los factores que explican las diferencias entre hombres y mujeres.

\section{Medida de la segregación}

En este trabajo se presentan mediciones de segregación laboral por medio del índice de disimilitud de Duncan (ID) (Duncan y Duncan, 1955). El cual mide el promedio de la magnitud de las diferencias entre la situación observada en determinado grupo de la fuerza laboral y un valor de referencia que se supone óptimo. Se calcula como:

$$
I D=\sum_{i}\left|h_{i}-m_{i}\right| / 2
$$

donde $h_{i}$ es el porcentaje de hombres trabajando en la ocupación $i$ y $m_{i}$ es el porcentaje de mujeres trabajando en la ocupación $i$. Este índice varía entre 0 y 1 , tomando el valor 0 cuando la distribución ocupacional de hombres y 
mujeres es idéntica, y 1, cuando hombres y mujeres no se superponen en ninguna ocupación, es decir, que hay segregación ocupacional perfecta. Este índice suele interpretarse como la proporción de mujeres (u hombres) ocupadas que sería necesario cambiar de ocupación para lograr la perfecta integración o distribución uniforme. El ID puede calcularse para tipos de ocupación o ramas de actividad siendo sensible al nivel de agregación de las categorías que se analizan. Esto es, a mayor agregación, menor es la segregación que se alcanza con el índice, pero no sólo el nivel de la misma puede diferir sino que también lo puede hacer su evolución en el tiempo así como la comparación entre áreas. ${ }^{3}$ Para analizar la evolución del ID es necesario considerar si los cambios que se verifican son estadísticamente significativos. Para ello se construyen intervalos de confianza utilizando la técnica de bootstraps, ${ }^{4}$ la cual permite estimar la distribución de un estadístico con base en el re-muestreo con reposición, a partir de la cual se construyen intervalos de confianza para ese estadístico.

\section{Medición de desajustes por calificaciones}

Los métodos estadísticos comúnmente usados para medir los desajustes por calificaciones se basan en la correspondencia efectivamente observada entre puestos de trabajo, ocupaciones y niveles de escolaridad respecto a las clasificaciones de las ocupaciones, a la media observada de la distribución de los ocupados (Verdugo y Verdugo, 1989) o al modo de dicha distribución. La información que se toma como referencia de la calificación de los trabajadores es el nivel o los años de escolaridad. Para conocer las características de los puestos de trabajo según los niveles de educación requeridos -en ausencia de encuestas específicas- suele utilizarse una clasificación de ocupaciones. Éstas suelen no estar actualizadas (Hartog, 2000), la conversión de las escalas de las clasificaciones en años de educación no es obvia (Halaby, 1994), se asume implícitamente que todos los trabajadores con un nivel de educación dado son perfectamente sustitutos y que hay requerimientos fijos de nivel de escolaridad

3 La mayor desagregación cuando el tamaño de la muestra disponible no es muy grande, debido a la falta de representatividad puede llevar a la aparición de ocupaciones con cero observaciones, en el total y, especialmente, en el caso de mujeres en ocupaciones mayoritariamente masculinas, distorsionando el nivel de segregación observado.

4 Los bootstraps calculados no tienen ningún supuesto previo sobre la distribución, corrigen por el sesgo en el valor medio estimado y se calculan para 95\% de confianza. Se realizaron 100 repeticiones de la muestra original. 
dentro de un mismo tipo de ocupación (Chevalier, 2003), aspectos éstos que debilitan la capacidad de análisis de los desajustes.

En este estudio se cuentan entre los sobrecalificados a los trabajadores que presentan más años de educación formal que las requeridas para el puesto que ocupan, según la CIUO885 y entre los subcalificados a los que cuentan con menos años de escolaridad que la requerida en dicha clasificación. La imputación de niveles de calificación y competencias a las ocupaciones (CIUO88) hace posible contrastar las calificaciones que requerirían los puestos de trabajo y las que poseen quienes los ocupan. Se trata pues del uso de un criterio normativo, es decir, basado en requerimientos formales preestablecidos. Espino (2011) señala que este método permite un análisis más adecuado para el caso uruguayo que el de la media o el modo, dado que la distribución observada de las calificaciones entre los trabajadores no aconsejaría el uso de esas medidas como referencias válidas. Por su parte, el valor de referencia presenta cierta objetividad en la definición de los requerimientos y es independiente de las calificaciones efectivamente observadas para la fuerza de trabajo del mercado laboral del país. ${ }^{6}$

\section{Diferencias salariales y segregación}

A los efectos de incorporar la segregación de género y el sexo entre las variables explicativas de las diferencias salariales entre hombres y mujeres se sigue la metodología propuesta por Bayard et al. (2003). Para ello se asume que esas

5 La Clasificación Internacional Uniforme de Ocupaciones (CIUO88) se usa desde el año 2000 por el INE para caracterizar los tipos de tareas que realizan los ocupados en Uruguay. Utiliza cuatro niveles o grupos de competencias definidas según las categorías y niveles de la Clasificación Internacional Normalizada de la Enseñanza (CINE). Los niveles de competencia no se especifican dentro de las grandes áreas ocupacionales, pero están directamente asociados con los grandes grupos de ocupaciones. Ocho de los grandes grupos de ocupaciones están asociados directamente a un nivel de competencias; por ejemplo, el grupo de ocupaciones científicas y profesionales requiere del nivel de competencias más elevado y el grupo de ocupaciones no calificadas requiere el nivel de competencias más bajo.

6 De manera similar a lo ocurrido en otros estudios, para Uruguay los resultados cuando se sigue el método de la media si bien mantienen los resultados en términos comparativos, las magnitudes son muy diferentes ya que casi la tres cuartas partes de los y las ocupadas resulta adecuadamente calificada; cuando se analiza a partir de la moda pasa lo mismo con los valores relativos pero las magnitudes se acercan algo más al método normativo (Espino, 2011). Para una discusión sobre esto véase G. Quintini (2011). 
diferencias son una función de las características individuales de capital humano y las de feminización del ámbito laboral de cada uno de los trabajadores, representada por el porcentaje de mujeres en cada ocupación, rama y tamaño de establecimiento.

De esta forma se plantea la ecuación salarial (1):

$l w_{p o i j}=\alpha+\beta$ sexo $_{p}+\gamma \operatorname{seg}_{o}+\delta$ segrama $_{i}+\lambda \operatorname{segtam}_{j}+X_{\text {poij }} \theta^{\prime}+\varepsilon_{\text {poij }}$

donde $l w_{p o i j}$ es el logaritmo del salario por hora que se explica por una variable dummy igual a 1 si el individuo $p$ es mujer ( 0 si es hombre), segM es el porcentaje de mujeres en la ocupación o, segrama es el porcentaje de mujeres por rama de actividad a un dígito $i$, segtam es el porcentaje de mujeres según tamaño del establecimiento $j . X$ representa un vector de variables individuales; $\varepsilon$ es un término de error bien comportado. $\beta$ sexo sería la diferencia atribuible al sexo de los individuos luego de controlar por la proporción de mujeres en las diferentes ocupaciones, ramas de actividad y categorías de establecimiento. Se puede interpretar como una medida de la discriminación, pero también capta diferencias en características no observables de los individuos, que en caso de afectar negativamente el salario estando más concentrada entre las mujeres que entre los hombres, su efecto se captura por este parámetro. Si el signo del parámetro es positivo, se puede interpretar que se debe a que hay discriminación hacia las mujeres y si es negativo, denota la situación inversa. El coeficiente $\theta$ representa la remuneración de las características del individuo. Si éstas son valoradas como positivas en el mercado laboral o implican una mayor productividad, cuanto mayor sea su valor, más será el salario y se traduce en un valor positivo del parámetro, si esas características se valoran negativamente o se vinculan con una menor productividad, será negativo. El parámetro $\gamma$ mide cómo influye la proporción de mujeres en las diferentes ocupaciones en el salario percibido. Un signo positivo en el parámetro supone que a mayor peso de las mujeres en la ocupación, mayor será el salario; análogamente se interpretan $\lambda$ y $\delta$.

La ecuación (1) según Bayard et al. (2003) se puede descomponer expresando las diferencias en promedio de los logaritmos de los salarios entre hombres y mujeres como en (2):

$$
\begin{aligned}
l w_{h}-l w_{m}= & \beta^{\prime}+\left(X_{h}-X_{m}\right) \theta^{\prime}+\gamma^{\prime}\left(\operatorname{seg}_{h}-\operatorname{seg}_{m}\right)+ \\
& \delta\left(\operatorname{segtam}_{h}-\operatorname{segtam}_{m}\right)+\lambda\left(\text { segrama }_{h}-\operatorname{segrama}_{m}\right)
\end{aligned}
$$


donde la prima en los coeficientes indica los valores estimados y los subíndices $m \mathrm{y} h$ en las variables indican el promedio para las mujeres y los hombres, respectivamente.

La diferencia promedio de los salarios se compone de cinco términos. La diferencia $X_{h}-X_{m}$ refleja las desemejanzas en las características de los individuos. La forma en que son remuneradas esas características en el mercado laboral está definida por el valor y el signo de $\theta$. La diferencia $\operatorname{seg} M_{h}-\operatorname{seg} M_{m}$ mide la contribución de la segregación ocupacional a la brecha salarial. Si las mujeres se concentran en pocas ocupaciones la diferencia entre ambos términos será negativa. Más aún, cuando hay segregación laboral, dado que las ocupaciones en que hay mayor proporción de mujeres están peor remuneradas, esta contribuiría al aumento de la brecha salarial (el término es positivo). La misma interpretación se puede dar al tercer y cuarto término. ${ }^{7}$

\section{Diferencias salariales y desajustes por calificación}

En este trabajo se estiman tres ecuaciones salariales (Badillo Amador y Vila Lladosa, 2005; Johansson y Katz, 2007). La primera ecuación salarial (Verdugo y Verdugo, 1989) procura analizar si el desajuste educativo con relación a la media de los ocupados explica las diferencias salariales entre trabajadores que tienen similares características y, por tanto, el mismo nivel educativo:

${\text { In } \text { salario }_{i}}_{=} \beta_{0}+\beta_{1}$ sobrecalif $_{i}+\beta_{2}$ subcalif $_{i}+\beta_{3} X_{i}+m_{i}$

donde la variable dependiente es el logaritmo del salario medio por hora del individuo $i$; sobrecalif $i$ es una variable dicotómica que adquiere valor 1 si el trabajador $i$ está sobreeducado en su empleo y 0 si no lo está; subcalif $_{i}$ es una variable dicotómica que vale 1 si el trabajador $i$ está infraeducado y 0 en caso contrario; $X_{i}$ es un vector de variables explicativas que recoge las características del individuo $i m_{i}$ es el término de perturbación aleatoria. La segunda y terce-

7 El análisis de las diferencias salariales por sexo controlando por segregación a lo largo de todas esas dimensiones tanto como otras características capturadas en $\beta^{\prime}$ puede pensarse como una descomposición tradicional de R. Oaxaca (1973), pero en este caso, se impone como restricción que los coeficientes sean los mismos para mujeres y hombres. Es decir, la segregación femenina, por lo tanto, podría afectar tanto los salarios femeninos como los masculinos. 
ra ecuaciones salariales permiten analizar si este fenómeno laboral explica las diferencias en el rendimiento de los años de educación de los trabajadores y, por tanto, las discrepancias salariales entre trabajadores que se encuentran en un mismo empleo.

En primer lugar, se estima una ecuación de salarios donde se examina la hipótesis de que todos los años de educación formal tengan la misma tasa de retorno, lo cual ocurriría si dicho retorno dependiera solamente de la oferta de laboral, como asume la teoría de capital humano (Becker, 1962 y 1975; Schultz, 1961).

In salario $_{i}=\lambda_{0}+\lambda_{1} e d u_{i}+\lambda_{2} X i+p i$

Luego se estima una ecuación salarial similar a la propuesta por Duncan y Hoffman (1981) para examinar si los retornos a la educación dependen únicamente de la educación requerida en el puesto de trabajo, como argumenta la teoría de competencia por el empleo (Thurow, 1975):

In salario $_{i}=a_{0}+a_{1} e d u_{i}+a_{2}$ AñosSobre $_{i}+a_{3}$ AñosInfra $_{i}+a_{4} X_{i}+e_{i}$

donde los años totales de educación formal alcanzados por el trabajador $i\left(e d u_{i}\right)$ se desagregan en años de sobreeducación (añosobre ${ }_{i}$ ) o en años de infraeducación (añosinfra $a_{i}$ ) (Hartog 1997, 2000).

\section{RESULTADOS}

La información analizada es para 2010 y proviene de la Encuesta Continua de Hogares (ECH) que realiza el Instituto Nacional de Estadística (INE) ${ }^{8}$ En el cuadro 1 se presentan los valores promedio para hombres y mujeres en el tramo de edad entre 24 y 65 ańos, que se encuentran ocupados y reportan ingresos por trabajo en la categoría de ocupación de asalariados (públicos y privados) de cada una de las variables que se incorporan al análisis.

8 Anteriormente a 2006 se relevaba información solamente para las localidades de 5000 y más habitantes. 


\section{Alma Espino}

Cuadrol. Asalariados de 24 a 65 años (total del país)

\begin{tabular}{|c|c|c|}
\hline \multirow[t]{2}{*}{ Variable } & \multicolumn{2}{|c|}{ Año 2010} \\
\hline & Hombres & Mujeres \\
\hline Logaritmo del salario & 5.50 & 5.40 \\
\hline Edad & 41.40 & 41.60 \\
\hline Años de estudio & 9.70 & 11.0 \\
\hline Montevideo & 0.47 & 0.51 \\
\hline Interior & 0.53 & 0.49 \\
\hline \multicolumn{3}{|l|}{ Tamaño de la empresa } \\
\hline Pequeña & 14.00 & 27.60 \\
\hline Mediana & 11.30 & 7.80 \\
\hline \multirow[t]{2}{*}{ Grande } & 74.70 & 64.6 \\
\hline & 100.00 & 100.0 \\
\hline \multicolumn{3}{|l|}{ Rama } \\
\hline Agropecuaria y minería & 7.00 & 1.40 \\
\hline Industrias manufactureras & 17.50 & 8.80 \\
\hline Electricidad, gas y agua & 2.10 & 0.90 \\
\hline Construcción & 11.90 & 0.60 \\
\hline Comercio, restaurantes y hoteles & 18.10 & 15.90 \\
\hline Transportes y comunicaciones & 10.20 & 3.10 \\
\hline Servicios a empresas & 7.10 & 7.60 \\
\hline Servicios comunales, sociales y personales & 26.20 & 61.7 \\
\hline Asiste al sistema de enseñanza & 0.05 & 0.09 \\
\hline Trabaja a tiempo completo & 0.96 & 0.81 \\
\hline Experiencia laboral (en años) & 25.70 & 24.60 \\
\hline Tiempo en su empleo actual (en años) & 9.40 & 8.60 \\
\hline
\end{tabular}

Fuente: ECH-INE.

\section{Segregación laboral de género}

Los resultados del cálculo de la segregación laboral de género se presentan en el cuadro 2. La segregación ocupacional tendió a aumentar a lo largo de la década en el sector privado mientras disminuyó en el público. Amarante 
y Espino (2002) encuentran que la segregación ocupacional en el mercado laboral uruguayo es alta con un valor del ID de 0.568 en $1999,0.539$ entre los asalariados del sector público y 0.607 entre los del privado, aunque en este caso se trabaja con un mayor nivel de agregación. El iD presenta valores sustancialmente menores para la segregación medida por rama de actividad (a un dígito de la clasificación ciru adaptada a Uruguay), decreciente en el sector privado y significativamente menor en el público. La menor segregación en el sector público también se observa en otros estudios, lo cual obedecería a las formas de contratación o de ascensos (por ejemplo, concursos) que han permitido incluso que las mujeres incursionen en puestos de dirección y tengan una representación mayor en el nivel profesional, aunque pesan de manera significativa en las ocupaciones de la enseñanza. ${ }^{9}$

Cuadro 2. Índice de Duncan. * Asalariados de 24 a 65 años (total del país) ${ }^{\star \star}$

\begin{tabular}{|c|c|c|c|c|c|c|c|c|c|c|c|c|}
\hline & \multicolumn{6}{|c|}{2001} & \multicolumn{6}{|c|}{2010} \\
\hline & \multicolumn{2}{|c|}{ Total } & \multicolumn{2}{|c|}{ Privado } & \multicolumn{2}{|c|}{ Público } & \multicolumn{2}{|c|}{ Total } & \multicolumn{2}{|c|}{ Privado } & \multicolumn{2}{|c|}{ Público } \\
\hline $\begin{array}{l}\text { Ocupaciones } \\
\text { (tres dígitos) }\end{array}$ & \multicolumn{2}{|c|}{0.595} & \multicolumn{2}{|c|}{0.62} & \multicolumn{2}{|c|}{0.547} & \multicolumn{2}{|c|}{0.608} & \multicolumn{2}{|c|}{0.637} & \multicolumn{2}{|c|}{0.509} \\
\hline $\begin{array}{l}\text { Intervalo } \\
\text { de confianza }\end{array}$ & 0.583 & 0.606 & 0.606 & 0.634 & 0.521 & 0.573 & 0.600 & 0.616 & 0.628 & 0.647 & 0.492 & 0.526 \\
\hline $\begin{array}{l}\text { Rama de } \\
\text { actividad } \\
\text { (un dígito) }\end{array}$ & \multicolumn{2}{|c|}{0.351} & \multicolumn{2}{|c|}{0.441} & \multicolumn{2}{|c|}{0.138} & \multicolumn{2}{|c|}{0.354} & \multicolumn{2}{|c|}{0.409} & \multicolumn{2}{|c|}{0.132} \\
\hline $\begin{array}{l}\text { Intervalo } \\
\text { de confianza }\end{array}$ & 0.337 & 0.366 & 0.423 & 0.458 & 0.114 & 0.161 & 0.343 & 0.364 & 0.398 & 0.421 & 0.113 & 0.151 \\
\hline $\begin{array}{l}\text { Tamaño } \\
\text { de empresa }\end{array}$ & \multicolumn{2}{|c|}{0.155} & \multicolumn{2}{|c|}{0.205} & \multicolumn{2}{|c|}{$\mathrm{nc}$} & \multicolumn{2}{|c|}{0.135} & \multicolumn{2}{|c|}{0.190} & \multirow{2}{*}{\multicolumn{2}{|c|}{$\mathrm{nc}$}} \\
\hline $\begin{array}{l}\text { Intervalo } \\
\text { de confianza }\end{array}$ & 0.142 & 0.169 & 0.188 & 0.222 & & & 0.128 & 0.143 & 0.180 & 0.201 & & \\
\hline
\end{tabular}

*Tipo de ocupaciones a tres dígitos de la CIU088. **Calculado con boostrap.

Fuente: Elaboración propia con base en ECH-INE.

9 Esta menor segregación puede constituirse en un aspecto positivo, si los espacios ganados obedecen a una real ruptura de los esquemas tradicionales de asignación de puestos, pero también puede reflejar relaciones de desigualdad, en tanto los hombres no permanecen en el sector público dado las mejores remuneraciones que reciben en el sector privado, donde es mayor la segregación (Ibáñez Pascual, 2010). 
La disminución de la segregación laboral de género resulta compatible con la disminución observada en la brecha salarial por sexo en el periodo. En particular debe considerarse que entre los asalariados del sector público esa brecha ha tendido a cerrarse y en algunos periodos favorece a las mujeres.

\section{Desajustes por calificaciones}

La proporción de trabajadores asalariados según tipos de ocupación de acuerdo a la CIUO88 a tres dígitos afectados por sobre y subcalificación con respecto a los niveles educativos requeridos se muestra en el cuadro 3. Hay una tendencia a la disminución de la subcalificación para ambos sexos durante el periodo. Las mujeres presentan una menor proporción de subcalificación reflejando el mayor nivel educativo promedio de la fuerza de trabajo femenina. La sobrecalificación es un problema de mayor magnitud -afecta a una mayor proporción de la fuerza de trabajo en forma agregada- que el de la subcalificación y es creciente en el tiempo. El aumento de la incidencia de la sobreeducación en general pero en particular entre las mujeres, obedecería a las dificultades para acceder al empleo, que las estimulan a mejorar su situación educacional para poder aspirar a una mayor gama de oportunidades ("señalización"). La segregación -tanto vertical como horizontal de género- en condiciones de aumento de la tasa de actividad femenina, también podría contribuir a esta explicación. Es decir, independiente de la formación educativa, tienden a insertarse o ser contratadas preferentemente en ciertas ramas y tipos de ocupación (Espino, 2011).

\section{Efecto de la segregación y desajustes por calificación sobre las brechas salariales}

\section{Sexo, segregación y brechas salariales}

En el cuadro 4 se presentan los resultados de las estimaciones para la ecuación (1) que incorporan los diferentes grupos de variables. El coeficiente asociado al sexo femenino es negativo y aumenta su valor cuando se incluyen los controles de características individuales. Esto podría implicar que pese a la caída observada en las brechas salariales, el factor que podría atribuirse a la discriminación o como ha sido señalado, a variables no observables en la investigación, ha crecido como parte de la explicación de dichas brechas. Si bien la educación tiende a compensar las diferencias entre hombres y mujeres, 
como se ve, no lo logra completamente. El cuadro 4 muestra que al incluir las diferentes medidas de segregación laboral el coeficiente asociado al sexo del trabajador disminuye $34 \%$ en valor absoluto (de -0.269 a -0.178). En este sentido, se obtienen resultados similares a los de Bayard et al. (2003), o sea, que aunque una parte importante de la brecha se explica por la segregación ocupacional femenina por tipo de ocupación y sector económico, una parte sustancial de esta brecha continúa siendo atribuible al sexo de los individuos. Sin embargo, a diferencia de ese estudio, cuando se colocan las variables de control asociadas a las características individuales observables, la contribución del sexo aumenta y la educación y la trayectoria laboral solamente contrarrestan esos factores contribuyendo apenas a disminuir la magnitud de la brecha.

Cuadro 3. De 24 a 65 años proporción de trabajadores según calificación y características individuales de los asalariados (sectores público y privado)

\begin{tabular}{|c|c|c|c|c|c|c|}
\hline \multirow[b]{2}{*}{ Total de asalariados } & \multicolumn{3}{|c|}{2000} & \multicolumn{3}{|c|}{2010} \\
\hline & Sobre & Sub & Adecuado & Sobre & Sub & Adecuado \\
\hline Mujeres & 56.6 & 23.5 & 19.9 & 62.9 & 19.2 & 17.9 \\
\hline Hombres & 40.2 & 40.9 & 18.9 & 46.1 & 34.2 & 19.7 \\
\hline \multicolumn{7}{|c|}{ Asalariados del sector público } \\
\hline Mujeres & 69.2 & 18.3 & 12.5 & 71.1 & 15.1 & 13.9 \\
\hline Hombres & 45.2 & 36.1 & 18.7 & 54.0 & 27.5 & 18.5 \\
\hline \multicolumn{7}{|c|}{ Asalariados del sector privado } \\
\hline Mujeres & 52.3 & 25.3 & 22.4 & 60.3 & 20.5 & 19.2 \\
\hline Hombres & 38.3 & 42.7 & 19.0 & 43.9 & 36.1 & 20.1 \\
\hline \multicolumn{7}{|l|}{ Región } \\
\hline Interior & 39.9 & 37.9 & 22.3 & 48.3 & 30.1 & 21.6 \\
\hline Montevideo & 54.6 & 28.4 & 17.1 & 60.8 & 23.3 & 15.9 \\
\hline \multicolumn{7}{|l|}{ Edad } \\
\hline Hasta 29 años & 57.0 & 25.0 & 18.0 & 62.4 & 20.4 & 17.2 \\
\hline 30 a 39 años & 52.2 & 27.2 & 20.7 & 59.2 & 22.9 & 17.9 \\
\hline 40 a 49 años & 48.3 & 32.8 & 19.0 & 52.5 & 27.9 & 19.6 \\
\hline 50 y más & 36.2 & 44.3 & 19.5 & 46.0 & 33.9 & 20.1 \\
\hline Total & 48.0 & 32.7 & 19.4 & 54.4 & 26.8 & 18.8 \\
\hline
\end{tabular}

Fuente: Elaboración propia en base a ECH-NE. 


\section{Alma Espino}

Cuadro 4. Estimaciones del logaritmo del salario y proporción de mujeres en las ocupaciones, ramas de actividad y establecimientos. Trabajadores asalariados de 24 a 65 años (total del país, 2010)

\begin{tabular}{|c|c|c|c|c|}
\hline \multirow[t]{2}{*}{ Mujer } & $-0.143^{\star \star \star}$ & $-0.266^{\star \star \star}$ & $-0.269 * \star \star$ & $-0.179^{\star * \star}$ \\
\hline & $(0.00833)$ & $(0.00683)$ & $(0.00680)$ & $(0.00903)$ \\
\hline \multirow[t]{2}{*}{ Educación en años } & & $0.0886^{\star * \star}$ & $0.0895^{\star * *}$ & $0.0915^{\star \star *}$ \\
\hline & & $(0.00152)$ & $(0.00141)$ & $(0.00140)$ \\
\hline \multirow[t]{2}{*}{ Experiencia 1} & & $0.0186^{\star * \star}$ & $0.0148^{\star \star \star}$ & $0.0143^{\star * *}$ \\
\hline & & $(0.00157)$ & $(0.00152)$ & $(0.00151)$ \\
\hline \multirow[t]{2}{*}{ Experiencia 2} & & $-0.00014^{\star \star \star}$ & $-0.00015^{\star \star \star}$ & $-0.00013^{\star \star \star}$ \\
\hline & & $(2.22 \mathrm{e}-05)$ & $(2.18 \mathrm{e}-05)$ & $(2.17 \mathrm{e}-05)$ \\
\hline \multirow{2}{*}{$\begin{array}{l}\text { Tiempo de trabajo } \\
\text { en el empleo actual } 1\end{array}$} & & $0.0373^{\star \star \star}$ & $0.0355^{\star \star \star}$ & $0.0366^{\star \star \star}$ \\
\hline & & $(0.00107)$ & $(0.00109)$ & $(0.00109)$ \\
\hline \multirow{2}{*}{$\begin{array}{l}\text { Tiempo de trabajo } \\
\text { en el empleo actual } 2\end{array}$} & & $-0.000494^{\star \star \star}$ & $-0.00049 * * *$ & $-0.000523^{\star \star \star}$ \\
\hline & & $(3.30 \mathrm{e}-05)$ & $(3.34 \mathrm{e}-05)$ & $(3.34 \mathrm{e}-05)$ \\
\hline \multirow[t]{2}{*}{ Tiempo completo } & & $-0.326^{\star \star \star}$ & 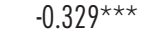 & $-0.367^{\star \star \star}$ \\
\hline & & $(0.0108)$ & $(0.0107)$ & $(0.0109)$ \\
\hline \multirow[t]{2}{*}{ Montevideo } & & & $0.101^{\star \star \star}$ & $0.105^{\star \star \star}$ \\
\hline & & & $(0.00781)$ & $(0.00775)$ \\
\hline \multirow[t]{2}{*}{ Sectores (público-privado) } & & & $0.0853^{\star * *}$ & $0.105^{\star \star \star}$ \\
\hline & & & $(0.00875)$ & $(0.00942)$ \\
\hline \multirow{2}{*}{$\begin{array}{l}\% \text { de mujeres } \\
\text { en las ocupaciones }\end{array}$} & & & & $-0.132^{\star \star \star}$ \\
\hline & & & & $(0.0146)$ \\
\hline \multirow{2}{*}{$\begin{array}{l}\text { \% de mujeres según rama } \\
\text { de actividad }\end{array}$} & & & & $-0.0694^{* * *}$ \\
\hline & & & & $(0.0232)$ \\
\hline \multirow{2}{*}{$\begin{array}{l}\text { \% proporción de mujeres por } \\
\text { tamaño de establecimiento }\end{array}$} & & & & $0.0104^{\star \star \star}$ \\
\hline & & & & $(0.00116)$ \\
\hline \multirow[t]{2}{*}{ Constante } & $6.301^{\star \star *}$ & $4.876^{\star * \star}$ & $4.608^{\star \star \star}$ & $4.728^{\star \star \star}$ \\
\hline & $(0.0281)$ & $(0.0511)$ & $(0.0520)$ & $(0.0522)$ \\
\hline Observaciones & 57194 & 57194 & 57194 & 57194 \\
\hline
\end{tabular}

Errores estándar entre paréntesis ${ }^{\star} p<0.1,{ }^{\star \star} p<0.05,{ }^{\star \star \star} p<0.01$. Fuente: ECH-INE. 
Cuadro 5. Contribuciones a la brecha salarial.

(1) Trabajadores asalariados de 24 a 65 años (total del país, 2010)

\begin{tabular}{|c|c|c|c|c|c|}
\hline & Hombres & Mujeres & (1)-(2) & $\begin{array}{c}\text { Contribución } \\
\text { absoluta }\end{array}$ & $\begin{array}{c}\text { Contribución } \\
\text { relativa }\end{array}$ \\
\hline & (1) & (2) & (3) & (4) & (5) \\
\hline $\begin{array}{l}\text { Logaritmo del salario } \\
\text { por hora }\end{array}$ & 5.49 & 5.37 & 0.119 & & \\
\hline Mujer & 0.00 & 1.00 & -1.00 & 0.18 & 141.8 \\
\hline Educación (en años) & 9.84 & 11.14 & -1.30 & -0.12 & -94.0 \\
\hline Experiencia 1 & 25.50 & 24.54 & 0.97 & 0.01 & 10.9 \\
\hline Experiencia 2 & 794.62 & 750.74 & 43.89 & -0.01 & -4.6 \\
\hline $\begin{array}{l}\text { Tiempo de trabajo } \\
\text { en el empleo actual } 1\end{array}$ & 9.63 & 8.76 & 0.87 & 0.03 & 25.1 \\
\hline $\begin{array}{l}\text { Tiempo de trabajo } \\
\text { en el empleo actual } 2\end{array}$ & 193.77 & 168.29 & 25.48 & -0.01 & -10.6 \\
\hline Tiempo completo & 0.96 & 0.82 & 0.14 & -0.05 & -41.7 \\
\hline Montevideo & 0.46 & 0.50 & -0.04 & 0.00 & -3.2 \\
\hline Sectores (público-privado) & 0.23 & 0.26 & -0.03 & 0.00 & -2.7 \\
\hline $\begin{array}{l}\text { \% de mujeres en las } \\
\text { ocupaciones }\end{array}$ & 0.26 & 0.74 & -0.48 & 0.06 & 50.4 \\
\hline $\begin{array}{l}\% \text { de mujeres según rama } \\
\text { de actividad }\end{array}$ & 0.41 & 0.58 & -0.17 & 0.03 & 21.8 \\
\hline $\begin{array}{l}\text { \% mujeres por tamaño de } \\
\text { establecimiento }\end{array}$ & 2.17 & 1.21 & 0.01 & 0.01 & 6.7 \\
\hline
\end{tabular}

Fuente: ECH-INE.

Los resultados mostrados en el cuadro 5 indican que la brecha es atribuible en orden decreciente al sexo de los trabajadores -que absorbe todo el aporte de la educación a la disminución de la brecha-, la segregación ocupacional 
$(50 \%)$ y por rama $(22 \%)^{10}$ (cuadro 5). Macpherson y Hirsch (1995) advierten que el porcentaje de mujeres en las ocupaciones puede ser una proxy para otras características de los puestos de trabajo por lo que los efectos negativos estimados sobre los salarios pueden parcialmente reflejar compensaciones a los diferenciales basados en preferencias de los trabajadores así como requerimientos de calificaciones. Pero también a los efectos de la interpretación de estos resultados debe recordarse que las mujeres están ubicadas en ocupaciones, ramas y establecimientos que tienen una alta proporción femenina. ${ }^{11}$ Los resultados presentados, confirman estudios anteriores dando cuenta de la importancia de la segregación laboral en la brecha salarial por sexo. Por otro lado, permiten observar que aunque una parte considerable de la brecha de sexo en los salarios se explica por la segregación de las mujeres en ocupaciones peor remuneradas y ramas con una porción sustancial de esta brecha sigue siendo atribuible al sexo de la persona.

\section{Sexo, segregación y desajustes}

Para investigar en qué medida los desajustes por calificaciones tienen un efecto en la brecha salarial de género se considerarán las variables de mismatch además de la educación y la segregación de género. Para ello se trabaja con cinco ecuaciones. En la primera, se evalúa la incidencia de la educación medida en años; en la segunda, se introducen las variables que corresponden a la proporción de ocupados sobrecalificados (sobre) y subcalificados (sub), respectivamente (Verdugo y Verdugo, 1989). En la tercera, sobre y subcalificación se miden en función de los años de escolaridad de los individuos respecto a los años requeridos, y en adelante se nombrará como sub y sobreeducación. Las variables añosub y añosob surgen de desagregar los años de educación reque-

10 A los efectos de la descomposición de las brechas se aplicó también el procedimiento de R. Oaxaca - con y sin corrección por sesgo de selección- considerando como estructura salarial no discriminatoria, la de los hombres. Los resultados son consistentes con los obtenidos por el anterior procedimiento y cuando se corrige por sesgo de selección éstos prácticamente no varían. El componente no explicado de las diferencias es positivo y significativo. Con respecto a los componentes de la parte explicada resalta nuevamente el coeficiente de los años de educación, y con respecto a la parte no explicada, los mayores coeficientes de signo positivo corresponden a la segregación ocupacional y por rama de actividad.

11 Bayard (2003) encuentra que en comparación con las estimaciones de una sola ecuación, el efecto total estimado de sexo de un individuo es del mismo tamaño en valor absoluto usando la estructura salarial de las mujeres, pero más grande si es la del salario masculino. 
ridos para cada tipo de ocupación, menos años de los requeridos o más. El parámetro de la variable "subeducados" debe ser interpretado como la prima salarial por cada ańo de educación de diferencia entre los requeridos y lo que se posee. El parámetro de "sobreeducados", como la reducción de los retornos a la educación para cada año de sobreeducación. En las ecuaciones (3') y (5') se agregan las variables de segregación laboral de género.

La estimación de la ecuación salarial (3) muestra, como era de esperar, que a mayor educación los trabajadores perciben mayores salarios mientras que el sexo -como ya se ha visto- tiene un fuerte efecto negativo (véase el cuadro 6). Las ecuaciones (4) y (5) muestran que en 2010, los años de subeducación tienen una tasa de retorno positiva $(7 \%)$, aunque inferior a la de los ańos requeridos en el puesto de trabajo (12\%); los ańos de sobreeducación tienen una tasa de retorno negativa (5\%). Estos resultados son consistentes con diversos estudios entre ellos (Hartog y Oostebeek, 1988; Rubb, 2003), en que los retornos a los ańos requeridos exceden a los que sobrepasan esos requerimientos, los cuales exceden (el valor absoluto de) los retornos a la menor cantidad de años respecto a los requeridos.

Como ha sido mencionado, la distribución de ocupaciones por género en el mercado laboral uruguayo permite constatar la presencia de una fuerte asociación de carácter estructural entre tipos de ocupación y género. A los efectos de relacionar estos resultados con la segregación laboral de género y la discriminación económica se incorpora la variable porcentaje de mujeres según tipo de ocupación, rama de actividad y tamaño del establecimiento. Al agregar estas variables disminuye el coeficiente asociado al sexo y se mantienen los asociados a los desajustes. Debe notarse que la variable de segregación ocupacional, y por ramas de actividad, presenta signo negativo dando cuenta de su contribución a la brecha.

Los resultados de las ecuaciones ( $\left.4^{\prime}\right)$ y (5') explican las diferencias salariales entre trabajadores que tienen similares características y los mismos años de educación según estén dentro del segmento de los sobre o subcalificados. Controlando por educación, los trabajadores sobrecalificados en promedio ganan $14.1 \%$ menos que los adecuadamente calificados y los subcalificados $13.2 \%$ por encima del promedio. La interpretación de esta situación podría consistir en que los sobrecalificados no tienen los niveles esperados de productividad; o la educación no la garantiza; o bien, que los bajos retornos salariales para los sobrecalificados estarían reflejando puestos con bajos salarios, o sea, concentrarse en ocupaciones de bajos salarios como podría ser el caso de las mujeres. Al agregar las variables de segregación disminuye ligeramente el coeficiente de la subcalificación. Los resultados coinciden con los presentados 
en la literatura sobre la temática (por ejemplo, Rubb, 2003): los retornos a la educación requerida son más altos que los retornos a la educación observada. Los años de sobre educación son recompensados, pero menos que los años de la educación requerida y los trabajadores con bajo grado educativo ganan menos que los trabajadores en trabajos similares con el nivel requerido de la educación. Sin embargo, aquellos ganan más que los trabajadores que tienen su mismo nivel de educación, pero en puestos de trabajo en los que el nivel requerido es menor. Esto sugiere que todos los años de educación formal no tendrían el mismo rendimiento educativo. Este dependería tanto de la educación ofertada como de la demandada en el empleo, como sugiere la teoría de la asignación (Hartog y Oosterbeek, 1988:188). El modelo ORU dado que brinda mayor información sobre la incidencia de las variables de capital humano en las remuneraciones, sería superior a las ecuaciones que sólo incluyen la educación efectivamente adquirida (Hartog, 2000).

A los efectos de estimar la incidencia de los desajustes en las brechas salariales entre los sexos se realizan nuevas descomposiciones. En el cuadro 7 se muestran las contribuciones absolutas y relativas de las variables asociadas a los desajustes por calificaciones. El encontrarse en el segmento de los sobre y subcalificados contribuye a la brecha salarial aunque en una magnitud algo menor que las variables de segregación.

Cuando se controla por las variables que dan cuenta de la escolaridad requerida por los puestos de trabajo, una escolaridad inferior o superior (todas medidas en años de educación) se obtiene que los resultados son similares a los anteriores (cuadro 8).

Las descomposiciones anteriores confirman hallazgos previos respecto a que las mujeres presentan retornos inferiores a los hombres (incluso cuando presentan los años requeridos para el puesto que ocupan). ${ }^{12}$ La mayor escolaridad femenina se ve contrarrestada por el menor rendimiento que presentan para las mujeres los años de educación que cumplen con lo requerido para el puesto de trabajo. A ello se agrega que al presentar una mayor sobreeducación se ven afectadas por la penalización salarial que ello representa. Los desajustes en las calificaciones pueden ser algunos de los mecanismos de "trato desigual" que aparece como "inexplicable" en descomposiciones tradicionales de la brecha salarial de género. A pesar de que las mujeres han invertido igual o más en educación que los hombres, sus salarios se ven deprimidos tanto porque los

12 Las regresiones realizadas en forma separada para hombres y mujeres comprueban esa afirmación. 
retornos son más bajos que para los hombres, como porque consiguen trabajos peor pagados que los hombres para el mismo nivel educativo.

Cuadro 6. Regresiones salariales. Trabajadores asalariados de 24 a 65 años (total del país, 2010)

\begin{tabular}{|c|c|c|c|c|c|}
\hline \multirow[t]{2}{*}{ Variables } & \multicolumn{5}{|c|}{ Coeficientes } \\
\hline & 3 & (3) & (4) & (5) & $\left(5^{\circ}\right)$ \\
\hline \multirow[t]{2}{*}{ Mujer (sexo) } & $-0.203^{\star \star \star}$ & $-0.158^{\star \star \star}$ & $-0.269^{\star \star \star \star}$ & $-0.243^{\star \star \star}$ & $-0.166^{\star \star \star}$ \\
\hline & $(0.00670)$ & $(0.00907)$ & $(0.00680)$ & $(0.00669)$ & $(0.00883)$ \\
\hline \multirow[t]{2}{*}{ Años de educación (Edu) } & $0.110^{\star \star \star}$ & $0.111^{\star \star \star}$ & $0.0895^{\star \star \star}$ & $0.122^{\star \star \star}$ & $0.123^{\star \star \star}$ \\
\hline & $(0.00157)$ & $(0.00157)$ & $(0.00141)$ & $(0.00161)$ & $(0.00160)$ \\
\hline \multirow[t]{2}{*}{ Sobrecalificación } & $-0.141^{\star \star \star}$ & $-0.143^{\star \star \star}$ & & & \\
\hline & $(0.00991)$ & $(0.00988)$ & & & \\
\hline \multirow[t]{2}{*}{ Subcalificación (Sub) } & $0.132^{\star \star \star}$ & 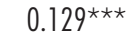 & & & \\
\hline & $(0.00999)$ & $(0.00998)$ & & & \\
\hline \multirow[t]{2}{*}{ Años requeridos (Añoreq) } & & & & $0.118^{\star \star \star}$ & $0.119^{\star \star \star}$ \\
\hline & & & & $(0.00139)$ & $(0.00139)$ \\
\hline \multirow{2}{*}{$\begin{array}{l}\text { Sobreeducación } \\
\text { (añossobre) }\end{array}$} & & & & $-0.0519^{\star \star \star}$ & $-0.0526^{\text {***}}$ \\
\hline & & & & $(0.00203)$ & $(0.00202)$ \\
\hline \multirow[t]{2}{*}{ Subeducación (añossub) } & & & & $0.0672^{\star \star \star}$ & $0.0657^{\star \star \star}$ \\
\hline & & & & $(0.00275)$ & $(0.00274)$ \\
\hline \multirow{2}{*}{$\begin{array}{l}\text { \% de mujeres en las } \\
\text { ocupaciones }\end{array}$} & & $-0.0423^{\star \star \star}$ & & & $-0.0948^{\star \star \star}$ \\
\hline & & $(0.0147)$ & & & $(0.0143)$ \\
\hline \multirow{2}{*}{$\begin{array}{l}\text { \% de mujeres por rama } \\
\text { de actividad }\end{array}$} & & $-0.103^{\star \star \star}$ & & & $-0.167^{\star \star \star}$ \\
\hline & & $(0.0228)$ & & & $(0.0223)$ \\
\hline \multirow{2}{*}{$\begin{array}{l}\text { \% de mujeres por } \\
\text { establecimiento }\end{array}$} & & $0.0102^{\star \star \star}$ & & & $0.0108^{\star \star \star}$ \\
\hline & & $(0.00132)$ & & & $(0.00128)$ \\
\hline \multirow[t]{2}{*}{ Constante } & $4.080^{\star \star \star}$ & $4.098^{\star \star \star}$ & $4.608^{\star \star \star}$ & $4.211^{\star \star \star}$ & $4.289^{\star \star \star}$ \\
\hline & $(0.0520)$ & $(0.0524)$ & $(0.0520)$ & $(0.0518)$ & $(0.0523)$ \\
\hline Observaciones & 57194 & 57194 & 57194 & 57194 & 57194 \\
\hline
\end{tabular}

Errores estándar entre paréntesis ${ }^{\star} p<0.1,{ }^{\star \star} p<0.05,{ }^{\star \star \star} p<0.01$.

Fuente: ECH-INE. 
Alma Espino

Cuadro 7. Contribuciones a la brecha salarial. Trabajadores asalariados de 24 a 65 años (total del país, 2010)

Hombres Mujeres Diferencia $\begin{gathered}\text { Contribución } \\ \text { absoluta }\end{gathered} \begin{gathered}\text { Contribución } \\ \text { relativa }\end{gathered}$

Logaritmo del salario por hora

\begin{tabular}{|c|c|c|c|c|c|}
\hline Sexo & 0.00 & 1.00 & -1.00 & 0.17 & 138.1 \\
\hline Educación (años) & 9.84 & 11.14 & -1.30 & -0.14 & -114.8 \\
\hline Experiencia 1 & 25.50 & 24.54 & 0.97 & 0.01 & 10.7 \\
\hline Experiencia 2 & 794.62 & 750.74 & 43.89 & -0.01 & -5.4 \\
\hline $\begin{array}{l}\text { Tiempo de trabajo } \\
\text { en el empleo actual } 1\end{array}$ & 9.63 & 8.76 & 0.87 & 0.03 & 25.4 \\
\hline $\begin{array}{l}\text { Tiempo de trabajo } \\
\text { en el empleo actual } 2\end{array}$ & 193.77 & 168.29 & 25.48 & -0.01 & -10.5 \\
\hline Tiempo completo & 0.96 & 0.82 & 0.14 & -0.05 & -41.3 \\
\hline Montevideo & 0.46 & 0.50 & -0.04 & 0.00 & -3.3 \\
\hline Sector público & 0.23 & 0.26 & -0.03 & 0.00 & -2.41 \\
\hline Sobrecalificación & 0.47 & 0.64 & -0.59 & 0.02 & 17.3 \\
\hline Subcalificación & 0.34 & 0.19 & 0.46 & 0.02 & 16.7 \\
\hline $\begin{array}{l}\% \text { de mujeres } \\
\text { en las ocupaciones }\end{array}$ & 0.26 & 0.74 & -0.48 & 0.05 & 38.1 \\
\hline $\begin{array}{l}\% \text { de mujeres por rama } \\
\text { de actividad }\end{array}$ & 0.41 & 0.58 & -0.17 & 0.03 & 23.8 \\
\hline $\begin{array}{l}\text { \% de mujeres por tamaño } \\
\text { de establecimiento }\end{array}$ & 2.17 & 1.21 & 0.97 & 0.01 & 7.6 \\
\hline
\end{tabular}

Fuente: ECH-NE. 
Cuadro 8. Contribuciones a la brecha salarial. Trabajadores asalariados de 24 a 65 años (2010)

Hombres Mujeres Diferencia $\begin{gathered}\text { Contribución } \\ \text { absoluta }\end{gathered} \begin{gathered}\text { Contribución } \\ \text { relativa }\end{gathered}$

\begin{tabular}{|c|c|c|c|c|c|}
\hline Logaritmo del salario por hor & & & & & \\
\hline Sexo & 0 & 1.00 & -1.00 & 0.166 & 136.1 \\
\hline Educación & 9.8 & 11.14 & -1.30 & -0.16 & -130.8 \\
\hline Experiencia 1 & 25.5 & 24.54 & 0.97 & 0.01 & 11.1 \\
\hline Experiencia 2 & 794.6 & 750.74 & 43.89 & -0.01 & -6.0 \\
\hline $\begin{array}{l}\text { Tiempo de trabajo } \\
\text { en el empleo actual } 1\end{array}$ & 9.6 & 8.76 & 0.87 & 0.03 & 24.8 \\
\hline $\begin{array}{l}\text { Tiempo de trabajo } \\
\text { en el empleo actual } 2\end{array}$ & 193.8 & 168.29 & 25.48 & -0.01 & -10.2 \\
\hline Tiempo completo & 0.96 & 0.82 & 0.14 & -0.05 & -39.9 \\
\hline Montevideo & 0.46 & 0.50 & -0.04 & 0.00 & -3.4 \\
\hline Sector público & 0.23 & 0.26 & -0.03 & -0.002 & -1.8 \\
\hline Años requeridos (Añosreq) & 9.3 & 9,57 & $-0,24$ & $-0,029$ & $-0,31$ \\
\hline Sobreeducación (añosobre) & 1.4 & 2.02 & -0.59 & 0.03 & 25.6 \\
\hline Subeducación (añosub) & 0.91 & 0.45 & 0.46 & 0.03 & 24.9 \\
\hline $\begin{array}{l}\% \text { de mujeres } \\
\text { en las ocupaciones }\end{array}$ & 0.26 & 0.74 & -0.48 & 0.046 & 37.5 \\
\hline $\begin{array}{l}\text { \% de mujeres por rama } \\
\text { de actividad }\end{array}$ & 0.41 & 0.58 & -0.17 & 0.029 & 23.5 \\
\hline $\begin{array}{l}\text { \% de mujeres por tamaño } \\
\text { de establecimiento }\end{array}$ & 2.1 & 1.2 & 0.97 & 0.010 & 8.6 \\
\hline
\end{tabular}

Fuente: ECH-INE. 


\section{CONCLUSIONES}

Este trabajo se propuso por una parte, reexaminar la magnitud de la segregación laboral de género y los desajustes por calificaciones y su incidencia en las brechas salariales. Los resultados indican que la segregación laboral de género resulta un fenómeno importante en Uruguay para entender la persistencia de las brechas salariales entre trabajadores de ambos sexos. A pesar de ello, aunque una parte considerable de la brecha de sexo en los salarios se explica por la segregación de las mujeres por ocupaciones y sectores con bajas remuneraciones, otra parte sustancial de esta brecha sigue siendo atribuible al sexo de las personas. La investigación -así como la discusión política- sobre la igualdad de género en los salarios ha prestado mayor atención a la segregación laboral que a las discrepancias entre la escolaridad y el nivel de empleo. Sin embargo, la probabilidad de encontrarse en situación de sobrecalificación es mayor para las mujeres y contrariamente, la de estar subcalificado es mayor para los hombres. La segregación ocupacional incide positivamente tanto entre los hombres como las mujeres en la probabilidad de la sobrecalificación. $\mathrm{Al}$ igual que en otros países, las mujeres presentan, contrario a los hombres, una educación que es superior a lo que normalmente se requiere para su ocupación, lo cual, contribuye a la brecha salarial de género. Es decir, las primas por educación son inferiores para las mujeres y a ello se agregan los efectos de los desajustes en la brecha salarial, entre los que se cuenta la penalización por la sobreeducación, situación que las afecta en mayor medida. Las brechas salariales son una expresión de la desigualdad de género vinculada a una variedad de factores propios y ajenos al mercado. El enfoque de capital humano estrictamente no permite explicar por qué, si las mujeres en la fuerza laboral presentan mayor escolaridad en promedio, y como se ha visto las diferencias de género en la experiencia de trabajo son pequeñas, se mantienen dichas brechas. Esto es, las variables tradicionales de capital humano prácticamente no explican la brecha salarial de género. De hecho, con un modelo que incluye sólo la educación y la experiencia, el término de dotación resulta negativo en la descomposición. Por tanto, ello puede estar dando cuenta de otras formas de discriminación tanto por el lado de la oferta como de la demanda relacionadas, como se ha visto, con la segregación y las dificultades para obtener empleos de mayor calificación - pese a sus mayores niveles educativos en promedio. Estos hallazgos tienen evidentes implicaciones de política; si la segregación a lo largo de diferentes dimensiones da cuenta de la mayor parte de la brecha salarial por sexo, las políticas tendientes a la igualdad de oportunidades en el empleo y la promoción y la acción afirmativa serían fundamentales para 
el cierre de esta brecha. La carencia de un análisis que permita considerar la segregación ocupacional de género en las empresas no permite por el momento extraer conclusiones de política más específica probablemente ligadas a la negociación colectiva.

\section{DEFINICIÓN DE VARIABLES}

Logaritmo del salario:

Sexo:

Educación:

Experiencia potencial:

Asiste:

Región:

Tiempo en el empleo actual:

Tiempo completo

$\%$ de mujeres en la ocupación:

$\%$ de mujeres por tipo

de establecimiento:

$\%$ de mujeres por rama

de actividad

Sobrecalificación:

Subcalificación:

Sobreeducación:

Subeducación: se calcula sobre salario líquido por hora de la ocupación principal

Dummy asume el valor 1 si es mujer; 0 si es hombres años de escolaridad finalizados en el sistema formal (Edu)

edad del individuo menos los 6 años previos al ingreso al sistema de enseñanza (Exp y Exp2)

Dummy asume el valor 1 si el individuo asiste al sistema educativo (asiste).

Dummy asume valor 1 si el individuo reside Montevideo.

años en el empleo actual (Expfirm y Expfirm2).

Dummy que toma valor 1 para quienes trabajan 20 horas semanales o más en el empleo principal (full).

en base a las ocupaciones a tres dígitos (CIUO88) (segM);

se basa en la clasificación de las empresas según número de empleados (segtam);

se calcula a partir de la clasificación CIIU a un dígito (segram).

Dummy adquiere valor 1 si el trabajador está sobrecalificado para el puesto que ocupa (sobrecalificado)

Dummy que vale 1 si el trabajador está infra-educado para el puesto que ocupa (subcalificado)

cantidad de años de educación menos años requeridos por la ocupación $($ AñosSobre $=$ AñosEduc - AñosReq $)$

cantidad de años de educación requeridos menos años observados (AñosInfra = AñosReq - AñosEduc) 


\section{BIBLIOGRAFÍA}

Allen, James Patrick, y Rolf Van der Velden "Educational mismatches versus skill mismatches: effects on wages, job satisfaction, and on-the-job search", Oxford Economic Papers, vol. 53, núm. 3, Oxford University Press, julio de 2001, pp.434-452.

Amarante, Verónica, y Alma Espino, "La segregación ocupacional de género y las diferencias en las remuneraciones de los asalariados privados. Uruguay, 1990-2000", Desarrollo Económico. Revista de Ciencias Sociales, vol. 44, núm. 173, Buenos Aires, IDEs, abril-junio de 2004, pp. 109-129.

, "La evolución de la segregación laboral por sexo en Uruguay (1986-1999)", Revista de Economía, segunda época, vol. ix, núm. 1, Montevideo, Banco Central del Uruguay, mayo de 2002, pp. 165-188.

Badillo-Amador, Lourdes; Antonio García-Sánchez, y Luis Vila "Mismatches in the spanish labor market: Education vs. competence match", International Advances in Economic Research, Berlín, vol. 11, núm. 1, febrero de 2005, pp. 93-109.

Barraza Narváez, Nacira, "Discriminación salarial y segregación laboral por género en las áreas metropolitanas de Barranquilla, Cartagena y Ediciones", Uninorte Barranquilla, Colombia, serie documentos IEEc, núm.31, junio de 2010.

Bayard, Kimberly; Judith Hellerstein; David Neumark, y Kenneth Troske, "New evidence on sex segregation and sex differences in wages from matched employee-employer data", Journal of Labor Economics, Chicago, The University of Chicago Press, vol. 21, núm. 4, octubre de 2003, pp. 887-922.

Becker, Gary, "Investment in human capital: A theoretical analysis", Journal of Political Economy, Chicago, University of Chicago Press, vol. 70, núm. 5, octubre de 1962, p. 9.

Becker, Gary, Human capital: A theoretical and empirical analysis, with special reference to education, $2^{\text {da }}$ ed., Chicago, University of Chicago, 1975.

Bergmann, Barbara, "Occupational segregation, wages and profits when employers discriminate by race or sex", Eastern Economic Journal, Easton, Eastern Economic Association, vol. 1, núm. 2, abril-julio de 1974, pp. 103110.

Bernat Díaz, Luisa Fernanda, "Los hombres al trabajo y las mujeres en la casa: ¿es la segregación ocupacional otra explicación razonable de las diferencias salariales por sexo en Cali?", Borradores de Economia y Finanzas, Colombia, ed. Universidad ICESI vol. 1 fasc. 16, 2009. 
Blau, Francine, y Lawrence M. Kahn, "Swimming upstream: Trends in the gender wage differential in the 1980s", Journal of Labor Economics, vol. 15, núm. 1, Chicago, University of Chicago Press, enero de 1997, pp. $1-42$.

Büchel, Félix, y Harminder Battu, "The theory of differential overqualification: Does it work?”, discussion paper, series, núm. 511, Bonn, Institute for the Study of Labor, junio de 2002.

Cohn Elchanan y Ying Chu Ng, "Incidence and wage effects of overschooling and underschooling in Hong Kong", Economics of Education Review, Amsterdam, Elsevier, vol 9, num. 2, abril de 2000, pp. 159-168.

Daly, Mary C.; Félix Büchel, y Greg J. Duncan, "Premiums and penalties for surplus and deficit education. Evidence from the United States and Germany", Economics of Education Review, Amsterdam, Elsevier, vol. 19, núm. 2, abril de 2000, pp. 169-178.

Duncan, Otis Dudley, y Beverly Duncan, "A methodological analysis of segregation indexes", American Sociological Review, Washington, American Sociological Association, vol. 20, núm. 2, abril de 1955, pp. 210-217.

Duncan, Greg J., y Saul D. Hoffman, "The incidence and wage effects of overeducation", Economics of Education Review, Amsterdam, Elsevier, vol. 1, núm. 1, 1981, pp.75-86.

Espino, Alma, "Evaluación de los desajustes entre oferta y demanda laboral por calificaciones en el mercado laboral de Uruguay", Revista de Economía del Rosario, vol. 14, núm. 2, Colombia, Facultad de Economía-Universidad del Rosario, julio-diciembre de 2011, pp. 99-133.

Frank, Robert H., "Why women earn less: The theory and estimation of differential overqualification", American Economic Review, Pittsburgh, American Economic Association, vol. 68, núm. 3, 1978, pp. 360-373.

Groeneveld, S., y Joop Hartog, "Overeducation, wages and promotions within the firm", Labour Economics, Chicago, University of Chicago Press, vol. 11, núm. 6, Chicago, 2004, pp.701-714.

Groot, Wim, y Henriètte Maassen Van den Brink, "Overeducation in the labor market: A meta-analysis", Economics of Education Review, Amsterdam, Elsevier, vol. 19, núm. 2, 2000, pp.149-158.

Hartog, Joop, "Over-education and earnings: where are we, where should we go?”, Economics of Education Review, Amsterdam, Elsevier, vol. 19, núm. 2, 2000, pp.131-147.

Hartog, Joop y Hessel Oosterbeek, "Education, allocation and earnings in the Netherlands: Overschooling?", Economics of Education Review, Amsterdam, Elsevier, vol. 7, núm. 2, 1988, pp.185-94. 
Hertz, Tom; Ana Paula de la O Campos, y Alberto Zezza, "Wage inequality in international perspective: Effects of location, sector, and gender", ESA, working paper (8/08/2008), Roma, Italia, Food and Agriculture Organization of the United Nations, Agricultural and Development Economics Division (ESA).

Johansson, Mats, y Katarina Katz, "Wage differences between women and men in Sweden. The impact of skill mismatch", working paper núm. 13, Uppsala, Institute for Labour Market Policy Evaluation, 2007.

Johnson, George, y Gary Solon, "Estimates of the direct effects of comparable worth", American Economic Review, Pittsburgh, American Economic Association, vol. 76, núm. 5, 1986, pp. 1117-1125.

Leuven, Edwin, y Hessel Oosterbeek, "Overeducation and mismatch in the labor market”, IZA, discussion papers 5523, Bonn, Institute for the Study of Labor (IZA), marzo de 2011.

Macpherson, David, y Barry Hirsch, "Wages and gender composition - Why do womens jobs pay less", Journal of Labor Economics, Chicago, University of Chicago Press, vol. 13, núm. 3, 1995, pp. 426-471.

Ñopo, Hugo; Juan Pablo Atal, y Natalia Winder, "New century, old disparities. Gender and ethnic gaps in Latin America", discussion paper núm. 5085, Bonn, Institute for the Study of Labor, julio de 2010.

Oaxaca, Ronald, "Male-Female wage differentials in urban labour markets", International Economic Review, Oxford, Wiley-Blackwell, 14, 1973, pp. 693-704.

Polachek, Salomon, "Occupational segregation among women: Theory, evidence, and a prognosis", C. B. Lloyd; E. S. Andrews, y C. L. Gilroy, en Women in the labor market, Nueva York, Columbia University Press, 1979, pp. 137-157.

Rivas, Florencia, y Máximo Rossi, Discriminación salarial en el Uruguay 19911997, documento de trabajo núm. 07/00, 2000, Universidad de la República, Departamento de Economía-Facultad de Ciencias Sociales.

Rubb, Stephen, "Overeducation in the labor market: A comment and reanalysis of a meta-analysis", Economics of Education Review, Amsterdam, Elsevier, 22, núm. 6, 2003, pp.621-629.

Rumberger, Russell, "The impact of surplus schooling on productivity and earnings", Journal of Human Resources, Madison, University of Wisconsin Press, vol. 22, núm. 1, 1987, pp. 24-50.

Sattinger, Michael, "Assignment models of the distribution of earnings", Journal of Economic Literature, Pittsburgh, American Economic Association, vol. 3, núm. 2, 1993, pp.851-880. 
Sicherman, Nachum, "Overeducation in the labor market", Journal of Labor Economics, Chicago, University of Chicago Press, vol. 9, núm. 2, 1991, pp.101-102.

Schultz, Theodore, "Investment in human capital", The American Economic Review, Pittsburgh, American Economic Association, vol. 51, núm.1, marzo de 1961, pp. 1-17.

Thurow, Lester C., Generating inequality: Mechanisms of distribution in the U.S. economy, Nueva York, Basic Books, 1975.

Quintini, G., "Over-qualified or under-skilled: A review of existing literature”, OECD, Social, Employment and Migration, working papers, núm. 121, oECD Publishing. http://dx.doi.org/10.1787/5kg58j9d7b6d-en (2011).

Verdugo, Richard, y Naomi Verdugo, "The impact of surplus schooling on earnings: Some additional findings", Journal of Human Resources, Wisconsin, University of Wisconsin Press, vol. 24, núm. 4, 1989, pp. 629-643.

Vieira, C. José António; Ana Rute Cardoso, y Miguel Portela, "Recruitment and pay at the establishment level: Gender segregation and the wage gap in Portugal", IZA, discussion papers 789, Bonn, Institute for the Study of Labor (IZA), 2003. 
